\title{
Évaluation de la gravité potentielle des erreurs interceptées par la conciliation des traitements médicamenteux en unité d'hospitalisation de courte durée
}

\author{
Assessing the Severity of Potential Harm of Errors Intercepted by Medication Reconciliation \\ on Admission in an Emergency Care Unit
}

\author{
F. Chabod · B. Gourieux · E. Lambert-Kuhn · P.-A. Gayol $\cdot$ B. Michel $\cdot$ P. Bilbault \\ Reçu le 17 mai 2018 ; accepté le 25 août 2018 \\ (C) SFMU et Lavoisier SAS 2018
}

Résumé Introduction : La conciliation des traitements médicamenteux (CTM) à l'admission hospitalière permet en mode rétroactif d'intercepter puis de corriger des erreurs en comparant la prescription hospitalière initiale à la liste des médicaments pris à domicile par le patient ; cette liste ou ce bilan médicamenteux étant réalisé à partir du croisement de différentes sources. L'objectif de l'étude a été d'évaluer la gravité potentielle des erreurs interceptées par la CTM en unité d'hospitalisation de courte durée (UHCD).

Matériel et méthode : La gravité potentielle des erreurs interceptées a été évaluée à l'aide d'un algorithme clinique selon deux modalités : en considérant uniquement la période d'hospitalisation et sans considération de durée ; c'està-dire comme si l'erreur avait perduré au-delà de la sortie hospitalière du patient. Ces erreurs ont été positionnées selon cinq niveaux de gravité clinique potentielle : mineur, significatif, majeur, critique et catastrophique.

Résultats : La CTM réalisée auprès de 122 patients a permis d'identifier 461 erreurs. Les classes médicamenteuses majoritairement impliquées ont concerné les systèmes cardiovasculaire $(n=151,32 \%)$, nerveux $(n=103,22 \%)$ et digestif

F. Chabod $(\bowtie) \cdot$ B. Gourieux · E. Lambert-Kuhn · B. Michel Service pharmacie-stérilisation,

hôpitaux universitaires de Strasbourg,

1, place de 1'Hôpital, F-67091 Strasbourg cedex, France

e-mail : faustine.chabod@orange.fr

P.-A. Gayol · P. Bilbault

Service des urgences, hôpitaux universitaires de Strasbourg,

1, place de 1'Hôpital, F-67091 Strasbourg cedex, France

B. Michel

Faculté de pharmacie, EA 7396, université de Strasbourg,

74, route du Rhin, F-67401 Illkirch cedex, France

P. Bilbault

Faculté de médecine, université de Strasbourg,

4, rue Kirschleger, F-67000 Strasbourg, France
( $n=86,18 \%)$. Les principaux types d'erreurs ont été les omissions $(n=381,83 \%$,) et les erreurs de dose $(n=57$, $12 \%) .1,1 \%$ des erreurs auraient pu avoir des conséquences cliniques majeures pour les patients durant la période d'hospitalisation et $8,5 \%$ d'entre elles des conséquences potentiellement majeures ou critiques en considérant un horizon temporel se prolongeant au-delà de l'hospitalisation.

Discussion : L'appréciation de la gravité potentielle des erreurs médicamenteuses traduit l'intérêt clinique de la CTM. Cette évaluation a permis de révéler en UHCD une sinistralité évitée jusque-là méconnue par les équipes médicales.

Mots clés Impact clinique potentiel · Erreur médicamenteuse $\cdot$ Conciliation des traitements médicamenteux Prise en charge médicamenteuse Urgences

Abstract Background: Medication reconciliation (MR) at hospital admission retroactively allows the interception and correction of errors by comparing the initial hospital prescription with the patient's medication list; this list or best possible medication history being obtained by crossing various sources. This study aimed to assess the severity of potential harm of errors intercepted by MR on admission to emergency care unit.

Method: The potential severity of the intercepted errors was assessed using a clinical algorithm considering two ways: considering the hospital stay only and regardless of duration; as if the error remained unresolved post discharge from hospital. These errors were positioned by a five-point scale rating their potential to cause harm: minor, significant, major, critical and catastrophic.

Results: MR of 122 patients revealed 461 medications errors. The three most common classes involved were cardiovascular system $(N=151,32 \%)$, nervous system $(N=103,22 \%)$ 
and gastrointestinal $(N=86,18 \%)$ medications. The most common types of errors were omission $(N=381,83 \%)$ and incorrect dose $(N=57,12 \%)$. Among the errors intercepted, $1.1 \%$ had potential major clinical consequences if we considered the hospital stay and $8.5 \%$ were ranked major or critical if they remained unresolved post discharge from hospital. Conclusion: The assessment of the potential harm of medication errors reflects the clinical interest of the MR. This evaluation revealed a potential clinical impact in emergency care unit previously unknown by the medical teams.

Keywords Potential clinical impact - Medication error · Medication reconciliation - Pharmaceutical care . Emergency care unit

\section{Introduction}

Lors de la prise en charge d'un patient en établissement de santé, la rupture du traitement médicamenteux aux points de transition de son parcours résulte, dans la plupart des cas, d'informations incomplètes ou mal communiquées entre les professionnels de santé en ville et à l'hôpital [1]. L'admission, les transferts internes et la sortie sont considérés comme des points de rupture dans le parcours de soins du patient, en particulier s'il est âgé $[2,3]$. Pour améliorer la sécurité thérapeutique et la continuité du traitement médicamenteux aux points de transition, la conciliation des traitements médicamenteux (CTM), considérée comme un processus d'interception et de correction des erreurs médicamenteuses, est très largement préconisée [4]. La Haute Autorité de santé (HAS) coordonne notamment le projet Med'Rec (Medication Reconciliation) relatif à la sécurisation de la prescription médicamenteuse aux points de transition du parcours de soins [1]. Un projet complémentaire, dénommé Med'Rec Kappa a évalué l'impact clinique de la CTM. Ce projet a conduit au développement d'un outil de cotation, présenté sous la forme d'un algorithme clinique, évaluant la gravité potentielle des conséquences des erreurs médicamenteuses interceptées par la CTM [5]. À ce jour, aucune réglementation française ne contraint les établissements de santé à mettre en œuvre la CTM. Cependant, cette activité est reconnue par les critères de certification des établissements de santé ainsi que l'arrêté du 6 avril 2011 sur la prise en charge médicamenteuse et est fortement encouragée par le contrat d'amélioration de la qualité et de l'efficience des soins (Caqes) mis en place en 2018 [6,7].

L'erreur médicamenteuse est l'omission ou la réalisation non intentionnelle d'un acte relatif à un médicament, qui peut être à l'origine d'un risque ou d'un événement indésirable pour le patient. Par définition, elle est évitable, car elle manifeste ce qui aurait dû être fait au cours de la prise en charge thérapeu- tique médicamenteuse d'un patient $[5,8]$. Les erreurs médicamenteuses sont une cause importante de morbidité et de mortalité à l'hôpital et ont également un impact financier $[9,10]$.

Les professionnels des établissements de santé sont confrontés à un manque de fiabilité de la liste des médicaments pris par le patient à son domicile. Le bilan médicamenteux se définit comme étant la liste exhaustive et complète des médicaments pris par le patient. Il constitue la cible à atteindre pour les professionnels en charge des conciliations à l'hôpital et contribue à sécuriser la prescription qui constitue l'étape initiale de la prise en charge médicamenteuse du patient [11]. En 2011, une enquête de pratique a été réalisée au centre hospitalier de Lunéville, portant sur la qualité informationnelle de la liste des médicaments, obtenue soit par le personnel du service des urgences, soit par celui du service de soins dans lequel le patient était hospitalisé. Le pourcentage de lignes complètes de médicaments détectées aux urgences était de 55 versus $81 \%$ dans le service de soins d'aval $[11,12]$. Ce constat est en accord avec celui de Caglar et al. qui considèrent que les listes de médicaments établies aux urgences sont justes dans seulement $13 \%$ des cas [13]. Cet état des lieux a fortement contribué à convaincre la communauté médicale de l'intérêt d'étendre la CTM aux urgences et à l'ensemble des services de soins accueillant notamment des patients âgés. L'unité d'hospitalisation de courte durée (UHCD) est une porte d'entrée à l'hôpital et constitue un lieu de transit pour les patients nécessitant une surveillance de moins de 24 heures ou sans lit d'aval, avant leur admission dans les services hospitaliers. La stratégie des hôpitaux universitaires de Strasbourg est d'assurer le développement de la CTM dès l'admission du patient en UHCD. L'objectif de l'étude est de caractériser l'impact clinique de la gravité potentielle des erreurs médicamenteuses, interceptées par la CTM, chez les patients admis en UHCD.

\section{Matériel et méthode}

Les hôpitaux universitaires de Strasbourg ont une capacité de 2711 lits et places et ont compté 147204 passages aux urgences en 2016 [14]. Ils comportent deux UHCD : une sur le site de l'hôpital de Hautepierre (14 lits) et une sur le site du Nouvel Hôpital civil (18 lits).

À l'admission du patient hospitalisé, la CTM se déroule en cinq étapes $[15,16]$. Tout d'abord, une recherche active d'informations sur les traitements médicamenteux du patient est effectuée. Les sources d'informations utilisées sont multiples. Parmi elles figurent l'entretien avec le patient et son entourage, l'entretien avec les professionnels des soins de premier recours (médecin généraliste, pharmacien d'officine, infirmière à domicile) et les sources matérielles accessibles (documents professionnels, boîtes de médicaments, systèmes d'information). Le bilan médicamenteux est alors 
formalisé et comparé à la prescription d'admission par le pharmacien, ce dernier mettant en évidence d'éventuelles divergences. Puis, un entretien médecin-pharmacien permet d'échanger sur ces divergences, en vue de la bonne continuité des traitements, de l'évaluation des risques et de la contribution à la pertinence des traitements du patient. C'est lors de cette étape que le pharmacien entre en discussion avec le médecin et détermine, par exemple, si la divergence résulte d'une omission ou d'un d'arrêt volontaire de traitement. Enfin, des modifications de prescriptions sont réalisées par le médecin pour corriger les éventuelles divergences non intentionnelles détectées. Celles-ci sont considérées comme des erreurs médicamenteuses.

Durant notre étude, les bilans médicamenteux ont été réalisés par quatre externes en pharmacie, et les CTM ont été conduites par un pharmacien « superviseur » et un interne en pharmacie. Les critères de priorisation des patients pour la réalisation du bilan médicamenteux sont les mêmes sur les deux sites : patients restant hospitalisés, patients âgés et polymédiqués présentant de multiples comorbidités, prescription de médicaments à risque, informations disponibles limitées sur le traitement à l'admission ou sur demande médicale. Nous utilisons la liste des médicaments à risque, développée par Doerper et al., présentée dans le tableau 1 [17]. Tous les patients ayant bénéficié d'une CTM en octobre 2015 selon les critères de priorisation mentionnés précédemment et ayant révélé une erreur médicamenteuse potentielle ont été inclus dans l'étude. Notre travail caractérise la gravité des erreurs médicamenteuses en utilisant l'échelle de cotation développée par la Société française de pharmacie clinique (SFPC), comportant cinq niveaux : mineure (sans conséquence pour le patient), significative (requiert une surveillance accrue pour le patient, mais sans conséquence clinique pour lui), majeure (conséquences cliniques temporaires pour le patient, à l'origine d'une atteinte physique ou psychologique réversible), critique (conséquences cliniques permanentes pour le patient, à l'origine d'une atteinte physique ou psychologique permanente irréversible) et catastrophique (mise en jeu potentiel du pronostic vital ou décès du patient) [18]. Il faut noter que d'autres échelles de cotation des erreurs médicamenteuses existent, telles que la classification selon le NCCMERP (National Coordinating Council for Medication Error Reporting and Prevention), développée par les Anglo-Saxons [8].

L'impact clinique potentiel des erreurs médicamenteuses est défini par l'algorithme clinique développé par Doerper et al. dans la figure 1 [17]. Cet algorithme cote la gravité potentielle de l'erreur médicamenteuse selon l'échelle de la SFPC en s'appuyant sur la nature de l'erreur médicamenteuse (erreur de patient, de dose, de médicament ou de stratégie thérapeutique, de voie d'administration, de moment de prise, de durée de traitement ou omission) ainsi que sur le statut de médicament à haut risque. Si le médicament appartient à cette liste, le niveau de cotation est alors a minima significatif.

\begin{tabular}{|ll|}
\hline Tableau 1 Liste des médicaments considérés à haut risque \\
\hline Erreur par omission & Erreur de dose \\
\hline Antiagrégants plaquettaires & Antinéoplasiques per os \\
Antibiotiques en traitement & Antiagrégants plaquettaires \\
de longue durée & Antibiotiques en traitement \\
Antiépileptiques & de longue durée \\
Antituberculeux & Antiépileptiques \\
Antivitamines K & Antinéoplasiques per os \\
Bêtabloquants dans IC & Antirétroviraux \\
et post-IDM & Antituberculeux \\
Digitaliques & Antivitamines K \\
Héparines & Bêtabloquants dans IC \\
Immunosuppresseurs, & et post-IDM \\
inhibiteurs de la calcineurine & Biguanides \\
Insulines & Digitaliques \\
Quinidiniques & Héparines \\
& Immunosuppresseurs, \\
& inhibiteurs de la calcineurine \\
& Insulines \\
& Opiö̈des morphiniques \\
& Potassium per os \\
& Quinidiniques \\
& Sulfamides hypoglycémiants \\
& et glinides \\
\hline IC insuffisance cardiaque ; IDM : infarctus du myocarde \\
\end{tabular}

L'impact clinique potentiel est ensuite déterminé à l'aide des informations « patients » suivantes : âge, sexe, motif d'admission, antécédents médicaux et chirurgicaux, valeurs biologiques, médicaments associés et erreurs médicamenteuses identifiées. La gravité potentielle des erreurs médicamenteuses est évaluée en considérant la gravité maximale possible qui s'exprimerait immédiatement ou tardivement si aucun événement intercurrent ne corrigeait l'erreur médicamenteuse ou l'expression de son dommage. Ainsi, deux modalités sont évaluées : en considérant uniquement la durée totale d'hospitalisation (extraite du système d'information, en évaluant la durée d'hospitalisation en UHCD et dans le service aval) et sans considération de période donnée (niveau de gravité supérieur). La cotation est organisée au sein de l'équipe pharmaceutique. Un pharmacien et un interne en pharmacie réalisent de manière indépendante et en aveugle la cotation des erreurs médicamenteuses. Chacun des deux acteurs parcourt la CTM réalisée et le dossier médical du patient afin de coter la gravité potentielle de chaque erreur médicamenteuse selon l'algorithme clinique de Doerper et al. Préalablement à la cotation, un apprentissage a été réalisé à partir d'erreurs médicamenteuses afin de s'assurer de la maîtrise du maniement de l'algorithme clinique. Les résultats sont mis en commun et analysés : en cas de conclusion similaire, le résultat est enregistré, 


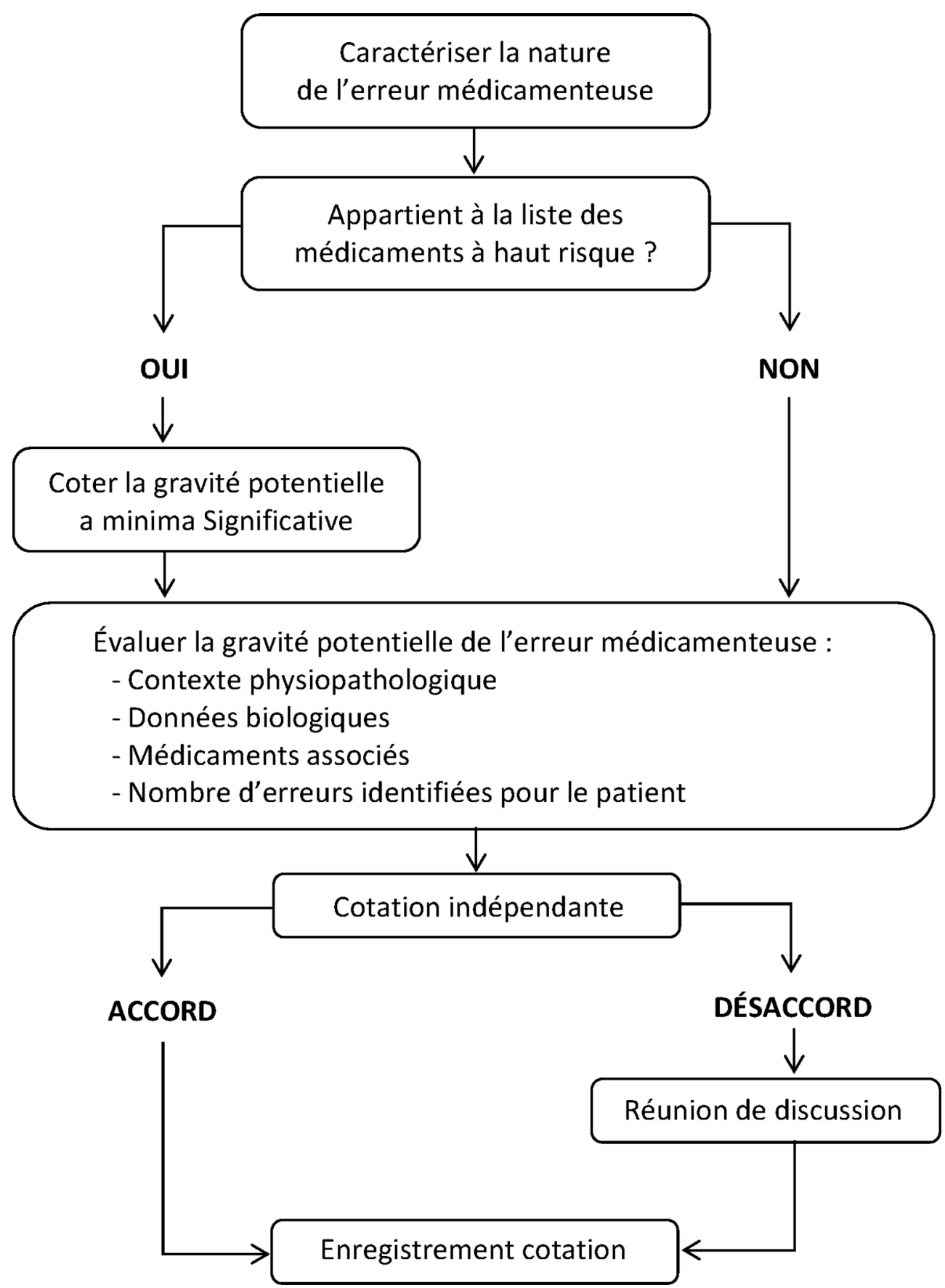

Fig. 1 Algorithme de cotation de la gravité potentielle des erreurs interceptées lors de la conciliation des traitements médicamenteux

et en cas de divergences, le dossier est étudié lors d'une réunion incluant deux médecins, un pharmacien et un interne en pharmacie. Cet échange permet d'obtenir un consensus pluridisciplinaire.

\section{Analyse statistique}

Les résultats sont présentés en valeur brute suivie de leur pourcentage.

\section{Résultats}

Au sein des hôpitaux universitaires de Strasbourg, $19 \%$ (136/709) des patients admis en UHCD durant l'étude ont été conciliés selon les critères de priorisation décrits précédemment. De plus, 90 \% (122/136) des CTM effectuées par les équipes pharmaceutiques durant notre étude ont conduit à enregistrer au moins une erreur médicamenteuse potentielle. L'âge moyen est de $79 \pm 11$ ans ( $\min : 41$; $\max$ : 97 ans), avec une proportion d'hommes de $51 \%$. Les motifs 
principaux d'admission sont les chutes $(n=24,20 \%)$, les motifs cardiologiques $(n=18,15 \%)$ et de la fièvre $(n=13$, $11 \%$ ). Parmi les 122 patients inclus dans l'étude, 62 ont été admis au Nouvel Hôpital civil et 60 à l'hôpital de Hautepierre. La durée moyenne de séjour des patients inclus est de 10,8 jours (min : 2 ; $\max : 150$ jours). Cette durée concerne l'ensemble du séjour du patient et est à distinguer de la durée moyenne d'hospitalisation en UHCD qui est de l'ordre de 24 à 48 heures.

Sur la population incluse, un total de 461 erreurs médicamenteuses potentielles a été enregistré, touchant les 122 patients, soit une moyenne de 3,8 erreurs potentielles par patient. Les types d'erreurs médicamenteuses les plus fréquents sont l'erreur par omission $(n=381,83 \%)$ suivie par l'erreur de dose $(n=57,12 \%)$ et les autres erreurs $(n=23,5 \%)$. Les classes médicamenteuses les plus impliquées sont les classes du système cardiovasculaire $(n=151$, $32 \%)$, du système nerveux $(n=103,22 \%)$, des voies digestives et du métabolisme $(n=86,18 \%)$. Les erreurs médicamenteuses ont été répertoriées plus précisément selon la sous-classe à laquelle elles appartiennent : les médicaments psycholeptiques sont les plus représentés $(n=56,12 \%$;), suivis des médicaments agissant sur le système rénineangiotensine $(n=30,6 \%)$ et des diurétiques $(n=30,6 \%)$ (Tableau 2). Dans $8 \%$ (38/461) des erreurs médicamenteuses potentielles, le médicament impliqué appartient à la liste des médicaments à haut risque. La sous-classe à risque des antithrombotiques est la plus impliquée $(n=19,49 \%)$, suivie des bêtabloquants $(n=6,15 \%)$ et des antiépileptiques $(n=5,13 \%)$.

Des exemples de cotation de la gravité potentielle des erreurs médicamenteuses sont décrits dans le tableau 3, distinguant l'expression clinique potentielle immédiate et tar-

Tableau 2 Sous-classes médicamenteuses les plus fréquemment impliquées dans les erreurs médicamenteuses

\begin{tabular}{|ll|}
\hline Sous-classes médicamenteuses & $\begin{array}{l}\text { Répartition, } \\
\boldsymbol{n}=\mathbf{4 6 1}(\mathbf{\%})\end{array}$ \\
\hline Psycholeptiques & $56(12)$ \\
Médicaments agissant sur le système & $30(7)$ \\
rénine-angiotensine & \\
Diurétiques & $30(7)$ \\
Bêtabloquants & $29(6)$ \\
Hypolipidémiants & $29(6)$ \\
Médicaments du diabète & $29(6)$ \\
Psychoanaleptiques & $25(5)$ \\
Système respiratoire & $25(5)$ \\
Médicaments contre les troubles & $22(5)$ \\
de l'acidité & \\
Antithrombotiques & $21(5)$ \\
Autres & $165(36)$ \\
\hline
\end{tabular}

dive. Il est à noter que les erreurs médicamenteuses potentielles révélées par notre étude ont été interceptées et corrigées par la CTM et n'ont eu aucune conséquence réelle pour les patients. Si l'on considère la durée totale d'hospitalisation, $1 \%(5 / 461)$ des erreurs médicamenteuses interceptées par la CTM auraient eu des conséquences cliniques potentielles temporaires (gravité majeure). En revanche, en considérant un horizon temporel se prolongeant au-delà de l'hospitalisation, $8 \%$ (39/461) d'entre elles auraient eu une conséquence clinique potentielle temporaire $(7 \%$, gravité majeure) ou permanente ( $1 \%$, gravité critique). Il n'y a eu aucune erreur médicamenteuse de gravité potentielle catastrophique. Les résultats sont présentés dans la figure 2.

Les 39 erreurs médicamenteuses de gravité potentielle majeure et critique ont été interceptées chez 32 patients. Ainsi, $26 \%$ des patients (32/122 patients) présentent une erreur de gravité potentielle cotée a minima majeure si l'on considère que l'erreur puisse perdurer après la sortie d'hospitalisation (Tableau 4). Pour ces 39 erreurs, celles par omission $(n=30,77 \%)$ sont les plus fréquentes, et la classe des antithrombotiques ( $n=12,31 \%$ ) est la plus impliquée, suivie des hormones systémiques $(n=5,13 \%)$ et des antiparkinsoniens $(n=5,13 \%)$. Pour $7 \%(8 / 122)$ des patients, la cotation indépendante a mis en évidence des résultats divergents entre le pharmacien et l'interne en pharmacie et a nécessité une délibération lors de la réunion de discussion médecin-pharmacien.

\section{Discussion}

L'évaluation de la gravité potentielle des erreurs médicamenteuses sur les deux UHCD des hôpitaux universitaires de Strasbourg a permis de révéler une sinistralité jusque-là méconnue par les équipes médicales. D'un point de vue méthodologique, la cotation de la gravité potentielle s'est déroulée de manière indépendante entre les évaluateurs, évitant qu'un intervenant impose sa notation ou influence le jugement de l'autre. En cas de résultats divergents, une réunion de discussion entre médecins et pharmaciens a permis de converger vers une cotation commune. Il est à noter qu'aucun n'a été impliqué dans la prise en charge du patient, dans un souci d'objectivité et pour obtenir une cotation à partir d'un niveau d'information homogène. Ces discussions permettent également des échanges d'opinions complémentaires sur l'appréciation de la gravité. Notons que seulement $7 \%$ des patients présentaient des résultats divergents lors de la cotation indépendante et ont été discutés en réunion. Ce faible chiffre peut s'expliquer par la phase d'apprentissage de l'outil de cotation, réalisée au préalable sur des patients tests.

Nous avons confronté nos résultats à ceux fournis par deux autres travaux ; l'étude de Quélennec et al. [19], incluant 256 patients de plus de 65 ans admis dans un service 
Tableau 3 Exemple de cotation de gravité potentielle des erreurs médicamenteuses

$\begin{array}{ll}\text { Exemple } & \begin{array}{l}\text { Cotation en considérant Cotation sans considérer } \\ \text { la durée d'hospitalisation de période }\end{array}\end{array}$

Un patient est admis pour fluctuation de l'état neurologique. Une prescription de clozapine $25 \mathrm{mg}, 1$ comprimé le soir, est réalisée par l'équipe médicale. La conciliation des traitements médicamenteux révèle que ce traitement a été arrêté récemment suite à des somnolences
Mineur (2 jours Significative

d'hospitalisation)

Majeure

Un patient est admis pour une suspicion d'accident ischémique transitoire.

L'omission de bimatoprost + timolol collyre, 1 goutte dans chaque œil le soir et brimonidine + timolol collyre 1 goutte dans chaque œil le matin, dans un contexte de glaucome primitif à angle ouvert est détectée

Un patient âgé de 79 ans est admis pour dyspnée brutale. L'omission de fluindione, 0,5 comprimé le soir, en prévention des complications thromboemboliques en rapport avec la fibrillation auriculaire est détectée

La conciliation des traitements médicamenteux d'une patiente de 71 ans, admise pour une cholécystite aiguë, ayant comme antécédent un diabète Majeure (5 jours d'hospitalisation)

Majeure (14 jours d'hospitalisation)

Critique

Significative (13
d'hospitalisation) de type 2 insulinorequérant, retrouve l'omission d'insuline glargine 100 unités/ml : 4 à 7 unités le soir en sous-cutané, selon glycémie

La prescription d'admission d'une patiente de 75 ans, admise pour un accident vasculaire cérébral ischémique est lévodopa + Majeure ( 9 jours d'hospitalisation)

\section{Critique}

Critique bensérazide 100/25 mg gélule : 1 à $8 \mathrm{~h}$ et 1 à $20 \mathrm{~h}$ (antécédent : maladie de Parkinson). La conciliation des traitements médicamenteux retrouve bien une prescription de lévodopa + bensérazide 100/25 mg, mais sous forme de gélule à libération prolongée à la posologie de 1 gélule à $8 \mathrm{~h}$, à $14 \mathrm{~h}$ et à $20 \mathrm{~h}$

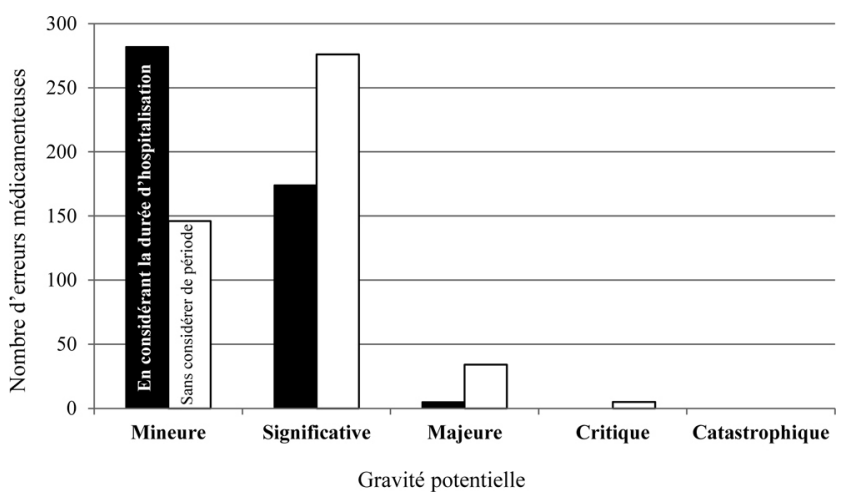

Fig. 2 Répartition des gravités potentielles des erreurs interceptées par la conciliation des traitements médicamenteux

de médecine interne et celle de Doerper et al., incluant 70 patients de plus de 65 ans issus de services de médecine et de chirurgie [17]. Notre étude révèle une moyenne de 3,8 erreurs médicamenteuses par patient, supérieure à celle rapportée par Quélennec et al. [19] (0,68 par patient) et Doerper et al. (2,1 par patient) [17]. Cela peut s'expliquer
Tableau 4 Gravité potentielle majeure et critique des erreurs interceptées par la conciliation des traitements médicamenteux

\begin{tabular}{|lll|}
\hline & $\begin{array}{l}\text { Évaluée } \\
\text { sur la durée } \\
\text { d'hospitalisation }\end{array}$ & $\begin{array}{l}\text { Évaluée sans } \\
\text { considération } \\
\text { de période }\end{array}$ \\
\hline Patients concernés & $2 / 122(2 \%)$ & $32 / 122(26 \%)$ \\
Erreurs & $5 / 461(1 \%)$ & $39 / 461(8 \%)$ \\
médicamenteuses & & \\
\hline
\end{tabular}

par le déclenchement de la CTM au plus proche de l'admission, par le caractère inattendu et non préparé d'un passage en UHCD, par la capitalisation d'informations identifiées par le personnel médical et soignant des services de médecine et de chirurgie ; ce qui atténue l'intérêt du déploiement de la CTM dans des services d'aval. Il faut noter que Quélennec et al. ont montré que $6 \%$ des erreurs médicamenteuses impliquent un médicament appartenant à la liste des médicaments à haut risque (contre $8 \%$ dans nos données) [19]. Cependant, les listes utilisées dans l'étude citée, ISMP 
(Institute for Safe Medication Practices) et NC NTI (North Carolina Narrow Therapeutic Index), diffèrent de celle adoptée pour notre travail $[20,21]$. Concernant la gravité potentielle des erreurs médicamenteuses, Quélennec et al. obtiennent, sur 173 erreurs médicamenteuses, $21 \%(n=36)$ d'erreurs de gravité significative et $6 \%(n=11)$ de gravité majeure, contre respectivement $38 \%(n=174)$ et $1 \%$ $(n=5)$ dans notre étude [19]. Ces divergences de résultats peuvent s'expliquer par le fait que Quélennec et al. n'utilisent pas la même échelle de cotation et prennent en compte uniquement la durée d'hospitalisation pour évaluer la gravité potentielle. Doerper et al. quant à eux identifient $17 \%$ $(n=12)$ de patients concernés par une erreur médicamenteuse de gravité potentielle a minima majeure si l'on considère la durée d'hospitalisation et $57 \%(n=40)$ si l'on considère que l'erreur perdure après la sortie d'hospitalisation (contre respectivement $2 \%[n=2]$ et $26 \%$ [ $n=32]$ dans notre étude) [17]. Nos résultats suivent la même tendance, à savoir que l'expression tardive est de gravité potentielle supérieure à l'expression immédiate.

Notre démarche apporte une vision nouvelle, car il n'existe aucune étude dans la littérature évaluant la gravité potentielle en UHCD selon deux modalités. Il est pourtant nécessaire de distinguer l'expression immédiate de l'expression tardive qui est de gravité potentielle supérieure. Notons que notre étude est menée en UHCD où la présence d'une équipe pharmaceutique dédiée à la $\mathrm{CTM}$, comme c'est le cas au centre hospitalier de Cambrai, est encore peu développée en France [22]. Pourtant, certaines situations telles que l'interception récemment publiée d'une interaction médicamenteuse potentiellement délétère entre le verapamil et l'ibrutinib, chez un patient admis aux urgences, montrent qu'il est primordial d'améliorer la sécurité des patients au point d'entrée de leur séjour hospitalier [23]. En UHCD, le pharmacien est généralement confronté à un manque de disponibilité des médecins en vue d'échanges sur les divergences identifiées par la CTM. Ce phénomène est accentué par la faible durée moyenne d'hospitalisation en UHCD et la non-reconduction systématique des traitements habituels non critiques, avec la présomption qu'ils le seront dans le service d'aval. De plus, la conciliation réalisée prématurément entre en concurrence avec les prescriptions d'admission à l'issue desquelles les divergences identifiées n'ont parfois plus cours. Ces éléments concourent à remettre en cause la mise en place de la CTM en UHCD. En revanche, la réalisation du bilan médicamenteux trouve sa place en UHCD à double titre. Elle est assimilée à un support pour la réalisation de la prescription d'admission par les médecins qui ont une connaissance partielle des médicaments pris par les patients du fait de l'hospitalisation non programmée. Le bilan médicamenteux réalisé précocement permet également d'initier le processus de CTM dès l'admission du patient dans le service d'aval. Dans une situation de ressources contraintes, la CTM est initiée sur la base de critères de priorisation qui ont été affinés grâce à ce travail. Elle est appliquée aux patients à risque (patients âgés polymédiqués et constituant une population complexe d'un point de vue médical) et aux médicaments à risque (antithrombotiques).

\section{Conclusion}

En UHCD, l'hospitalisation n'étant pas par définition programmée, les médecins sont confrontés à un réel défaut d'information sur les traitements habituels des patients. $\mathrm{La}$ réalisation de la CTM en UHCD, sous réserve de ressources pharmaceutiques dédiées à sa mise en œuvre et à sa pérennisation, réduit les erreurs médicamenteuses dès le début du parcours de soins du patient à l'hôpital et évite in fine une sinistralité d'origine médicamenteuse.

Conflits d'intérêts : les auteurs déclarent ne pas avoir de conflits d'intérêts.

\section{Références}

1. High 5s Project (2009) Action on patient safety. Assuring medication accuracy at transitions: medication reconciliation in care. http:// www.who.int/patientsafety/solutions/high5s/High5_overview.pdf (Dernier accès le 9 avril 2018)

2. Touraine M (2013) Ministère des Affaires sociales et de la Santé. Lettre ministérielle, 8 février 2013

3. Haute Autorité de santé (2013) Fiche points clés et solutions : comment réduire le risque de réhospitalisations évitables des personnes âgées ? http://www.has-sante.fr/portail/upload/docs/application/pdf/ 2013-06/fiche parcours_rehospitalisations_evitables_vf.pdf (Dernier accès le 9 avril 2018)

4. Institute for Healthcare Improvement (2011) How-to guide: prevent adverse drug events by implementing medication reconciliation. Cambridge, MA. http://app.ihi.org/LMS/Content/2cf9e482-3e914218-afe3-22f77b5025bc/Upload/HowtoGuidePreventADEs.pdf (Dernier accès le 18 juillet 2018)

5. Haute Autorité de santé (2015) Rapport d'expérimentation sur la mise en œuvre de la conciliation des traitements médicamenteux par neuf établissements de santé français. Annexe 5 : caractérisation de la gravité potentielle des conséquences de l'erreur médicamenteuse. http://www.has-sante.fr/portail/jcms/r_1498429/fr/linitiative-oms-high-5s (Dernier accès le 9 avril 2018)

6. Manuel de certification, V.2010, version janvier 2014, critère 20 (2014) « Prise en charge médicamenteuse du patient », E1.EA2. http://www.has-sante.fr/portail/jcms/c 1732464/fr/manuel-de-certification-des-etablissements-de-sante-v2010-edition-janvier-2014 (Dernier accès le 9 avril 2018)

7. Bertrand X (2011) Arrêté du 6 avril 2011 relatif au management de la qualité de la prise en charge médicamenteuse et aux médicaments dans les établissements de santé

8. National Coordinating Council for Medication Error Reporting and Prevention. What is a medication error? http://www.nccmerp.org/ about-medication-errors (Dernier accès le 9 avril 2018)

9. Basey AJ, Krska J, Kennedy TD, Mackridge AJ (2014) Prescribing errors on admission to hospital and their potential impact: a mixed-methods study. BMJ Qual Saf 1:17-25 
10. Karnon J, Campbell F, Czoski-Murray C (2009). Model-based cost-effectiveness analysis of interventions aimed at preventing medication error at hospital admission (medicines reconciliation). J Eval Clin Pract 2:299-306

11. Bonhomme J, Dony A, Baum T, et al (2013) La juste liste des médicaments à l'admission du patient hospitalisé : de la fiabilité des sources d'information. Risque et qualité 10:239-45

12. Doerper S, Morice S, Piney D, et al (2013) La conciliation des traitements médicamenteux : logigramme d'une démarche efficiente pour prévenir ou intercepter les erreurs médicamenteuses à l'admission du patient hospitalisé. Pham Hosp Clin 3:153-60

13. Caglar S, Henneman PL, Blank FS, et al (2011) Emergency department medication lists are not accurate. J Emerg Med 40:613-6

14. Hôpitaux universitaires de Strasbourg (2016) Chiffres clés. http:// www.chru-strasbourg.fr/sites/default/files/documents/chiffres_cles_2016.pdf (Dernier accès le 9 avril 2018)

15. Dufay E, Baum T, Doerper S, et al (2011) La conciliation des traitements médicamenteux : détecter, intercepter et corriger les erreurs médicamenteuses à l'admission des patients hospitalisés. Risques Qual 2:130-8

16. American Pharmacists Association, American Society of Health System Pharmacists (2012) Improving care transitions: optimizing medication reconciliation. J Am Pharm Assoc 52:e43-e52
17. Doerper S, Godet J, Alexandra JF, et al (2015) Development and multi-centre evaluation of a method for assessing the severity of potential harm of medication reconciliation errors at hospital admission in elderly. Eur J Intern Med 7:491-7

18. Société française de pharmacie clinique (2014) Le classeur de la revue des erreurs liées aux médicaments et dispositifs médicaux. http://www.sfpc.eu/fr/item1/summary/34-documents-sfpcpublic/465classeurdelaremedsfpc2014.html (Dernier accès le 9 avril 2018)

19. Quélennec B, Beretz L, Paya D, et al (2013) Potential clinical impact of medication discrepancies at hospital admission. Eur $\mathrm{J}$ Intern Med 6:530-5

20. Institute for Safe Medication Practices. Liste des médicaments à risque de l'ISMP. https://www.ismp.org/tools/highalertmedicationLists.asp (Dernier accès le 9 avril 2018)

21. North Carolina Board of Pharmacy. Liste des médicaments à risque du NC NTI (North Carolina Narrow Therapeutic Index). http:// ncbop.org/faqs/Pharmacist/faq_NTIDrugs.htm (Dernier accès le 9 avril 2018)

22. Schiettecatte S, Dambrine M, Bouche C, et al (2015) Activité de conciliation médicamenteuse aux urgences. Ann Fr Med Urg $5: 220-5$

23. Lambert Kuhn E, Levêque D, Lioure B, et al (2016) Adverse event potentially due to an interaction between ibrutinib and verapamil: a case report. J Clin Pharm Ther 41:104-5 